\title{
Numerical analysis of missile impact being shot by rocket propelled grenades with rod armour
}

\author{
T. Niezgoda, R. Panowicz, K. Sybilski \& W. Barnat \\ Department of Mechanics and Applied Computer Science \\ Military University of Technology, Poland
}

\begin{abstract}
During the operation of the UN and NATO forces in Africa and Asia it appeared that the equipment held and utilized by these organizations was not proof against the missiles being shot by rocket propelled grenades. The missiles utilized by the terrorist organizations belong to the first generation. They have a head with a shaped charge jet permitting them to pierce up to $300 \mathrm{~mm}$ RHA steel.

There are four protection methods against this hazard: reactive armour, thick proper armour, rod armour and active defence system. The reactive armour cannot be applied on each kind of vehicle. The thick, proper armour limits other important features of vehicles. The active defence systems continue to develop, except the Russian system "Arena". Therefore, the fast development of rod armour appeared. The main task of this armour type is to disturb the symmetry of the shaped charge jet as a result of the shock that is effective of the deflagration and not the forming of cumulative jet.

The finite element model of the missile with the cumulative head and its influence with two types of rod armour will be presented in this article. The cases of the missile impact with circular and square section for different typical speeds of missile will be considered.
\end{abstract}

Keywords: mechanics, finite element method, rods armour, RPG.

\section{Introduction}

As a result of armed conflicts observation all over the world and actions carried on by Polish army divisions within a framework of stabilization missions, we can notice a change in the way of fighting and the usage of available ammunition. It appears that currently the biggest threat is posed by two types of ammunition. 
The first are all kinds of landmines and improvised explosive device (IED), whose basic destructive factor is a pressure wave formed due to the explosion. The destruction effect is multiplied by all kinds of shrapnel (marbles, nails, screws etc.) placed inside or on the explosive device. The second group of the most threatening ammunition included are bullets with accumulation heads. They were worked out to destroy military armoured vehicles. Their popularity is connected, before all, with high penetration (reaching $900 \mathrm{~mm}$ of reinforced steel RHA [1]), low production cost and the simplicity of use.

High penetration of projectiles with an accumulation head is a big problem for the protection against this kind of ammunition. One of the possible protection methods against this threat is the use of rod armour. A perfect protection of this type should not allow the creation of accumulation stream, that is the striking power of a projectile with accumulation head. The principle of operation of this kind of armour consists in neutralizing the accumulation insert and the explosive material contained inside the projectile by a strong deformation of these elements. The result is an eventual creation of accumulation stream with a lower efficiency, in an extreme case - the lack of it. In this situation the destruction force is comparable with a detonation of an explosive material contained within the projectile and the reaction of fragments created by the explosion.

In the following article the results of a simulation of an impact of a projectile with an accumulation head type PG-7G in two different kinds of rod armours will be presented. Two impacts under different angles in respect to the projectile axis will be considered.

For the analysis LS-Dyna software was used containing an open implementation of finite elements method.

\section{Construction and principle of operation of projectile with an accumulation head}

A projectile with an accumulation head is made of three principle elements: head, rocket engine and flight stabilizer (fig. 1) $[2,3]$. The head contains the most important parts of the projectile: accumulation insert, explosive material, a shield and a fuse. During the shooting the projectile is fired outside the barrel towards the target and accelerates to the maximum speed reaching $300 \mathrm{~m} / \mathrm{s}$. In the moment of impact on the target the fuse is generating an electrical impulse exciting through the detonator of the explosive material. The effect of exciting the detonator is the detonation of the explosive material and creation of the accumulation stream.

The condition of achieving the maximum destruction power of the accumulation stream is a proper, very accurate workmanship of the device and the accumulation insert. In the case of both of these elements the inaccuracy of workmanship should not exceed $0,05 \mathrm{~mm}$, and the radius deviation should not be bigger than $0,03 \mathrm{~mm}$. Together with the increase of the array's inaccuracy its effectiveness decreases. In an extreme case, with large workmanship errors, an accumulation stream will not be created and the projectile's effect will be 


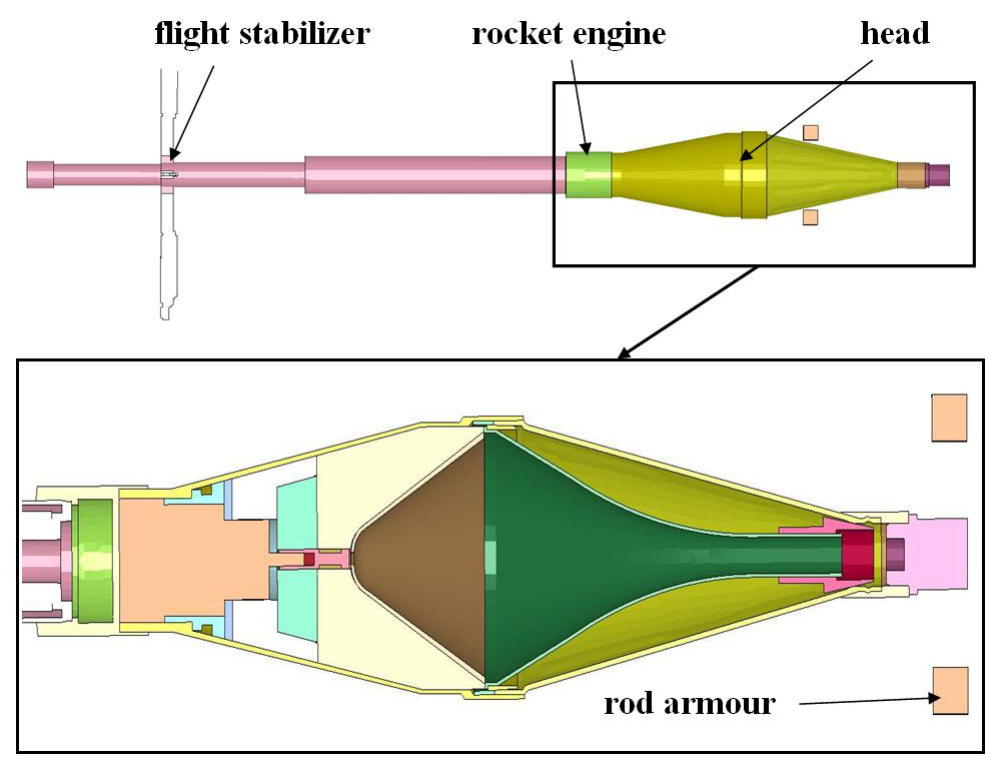

Figure 1: $\quad$ An intersection of the projectile PG-7G head.

comparable with the effects of an explosion of an explosive material with a mass of the charge contained in the head. This feature is used in rod armours. It is assumed, that with certain probability amounting to less than $50 \%$, it will not come into contact between the fuse of the incoming projectile and the elements of the armour. As a result an accumulation stream will not be created.

Results from the carried out analysis indicate that in most cases the projectile hits the rods with the elements of the projectile's casing, which causes deformation of the accumulation insert and crumbling of the explosive material, reducing considerably the effectiveness of this weapon.

\section{MES model construction of PG-7G projectile}

To carry out the projectile PG-7G type impact simulation in different kinds of rod armours the elaboration of a numerical model was necessary. The principal restriction during its construction was the maximal number of used finite elements. Together with the increase of the finite elements number the quantity of unknown in the dynamical equation increases, and with this the time needed to solve both this equation as well as the whole problem prolongs. To make the limitation of the number of finite elements possible, introducing certain simplification of the projectile's geometry was necessary (fig. 2), that do not influence the behaviour of the array as a whole. 
In the model five main simplifications were introduced:

1. Shoulders were removed and a hole in the tube between the nozzles and the fins.

2. The flight stabilizers were replaced by a cylinder with an identical mass.

3. Holes fastening the fins were removed.

4. Nozzles were removed.

5. A part of the fuse protruding outside the projectile was replaced with one cylinder.

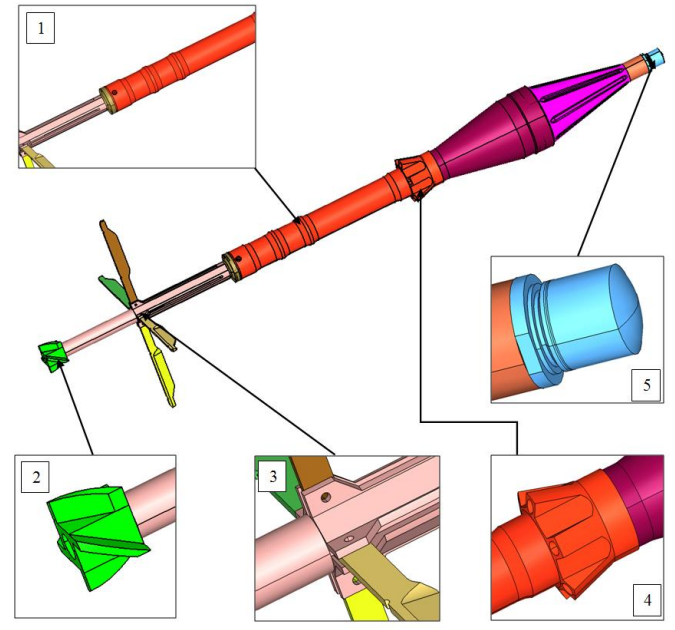

Figure 2: Projectile's geometry simplifications.

The numerical model constructed and used for the calculations is composed of 19 parts. Particular parts were attributed to material properties, that characterize the behaviour of real materials used in the analysed projectile. For the description of copper a simplified Johnson-Cook model was used. The behaviour of other materials was reflected by the use of a multilinear model (Piecewise_Linear_Plastic). This model allows to image materials characteristics by introducing an experimental curve displaying the dependence of stress from strain.

Between all of the parts of the model a contact was defined, that is usually used in dynamical problems for example in analysis of crash type. In these analyses, similarly as in described case, we deal with great deformations and elements displacement speeds. Parts of the projectile element are connected to each other with a screw thread. Between these elements of the model a contact, simulating this connection, was defined. The chosen contact type enables inserting a conditioned separation of connected elements.

During the simulation imaging of projectile PG-7G type impact into an obstruction made out of two angle sections, being a fragment of the rod armour structure, was endeavoured (fig. 3). In the first case rods with a circular intersection with a diameter of $15 \mathrm{~mm}$ were considered, and in the second - with 
a square intersection (side length $14 \mathrm{~mm}$ ). The rods dimensions were matched so that the mass of the armour in both solutions was close. In both cases it was accepted, that the total length of a singular rod amounted to $500 \mathrm{~mm}$. It was assumed, that the projectile hits centrally between the rods, at the half of their length.

The simulation was carried out for three initial speeds of the projectile $\mathrm{V}_{\mathrm{z}}$. In the first version the projectile moved with the minimal speed that it acquires during firing $(100 \mathrm{~m} / \mathrm{s})$, in the second with maximal speed $(300 \mathrm{~m} / \mathrm{s})$. The third case was put into practise for an intermediate speed amounting to $200 \mathrm{~m} / \mathrm{s}$.

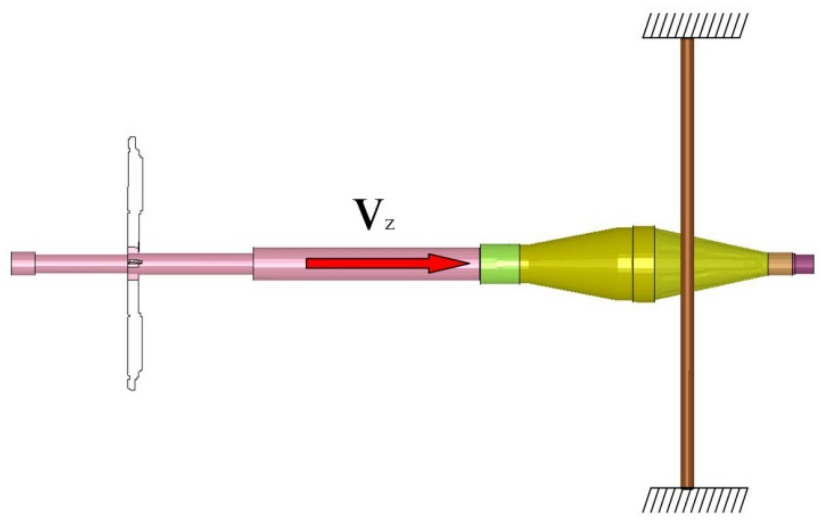

Figure 3: Initial-boundary conditions.

Numerical calculations were carried out in LS-Dyna system. This system is used, among others, for the analysis of fast-changing processes with the finite elements method. Based on the information contained in the model it builds a dynamical movement equation in the following form [4]:

$$
M \ddot{q}+C \dot{q}+K q=f
$$

This equation is solved then with the direct integration method (so called Explicite), that finds a wide use in the analysis of highly nonlinear phenomena (impacts, stamping, explosion wave influence on a structure). In calculations of this type we have to manage with great strains and strain speeds.

\section{Numerical analysis results}

In figures 4 and 5 the strain process of the projectile during the impact into the rod with a square intersection for the initial speeds amounting to 100 and 300 $\mathrm{m} / \mathrm{s}$ are presented. In the initial stage we can observe the crushing of the ballistic cap after the impact into the head's body. This impact causes strong folding of the body that in consequence results in significant damage of the explosive 
material. In the final stage it comes to the burring of the body and passage of the projectile between the rods. In attached figures one can notice that with the increase of the initial speed the way the projectile's elements behaviour changes. The head's body burring proceeds more on the outside of the projectile, the rods strain less, but the accumulation insert is strained more.
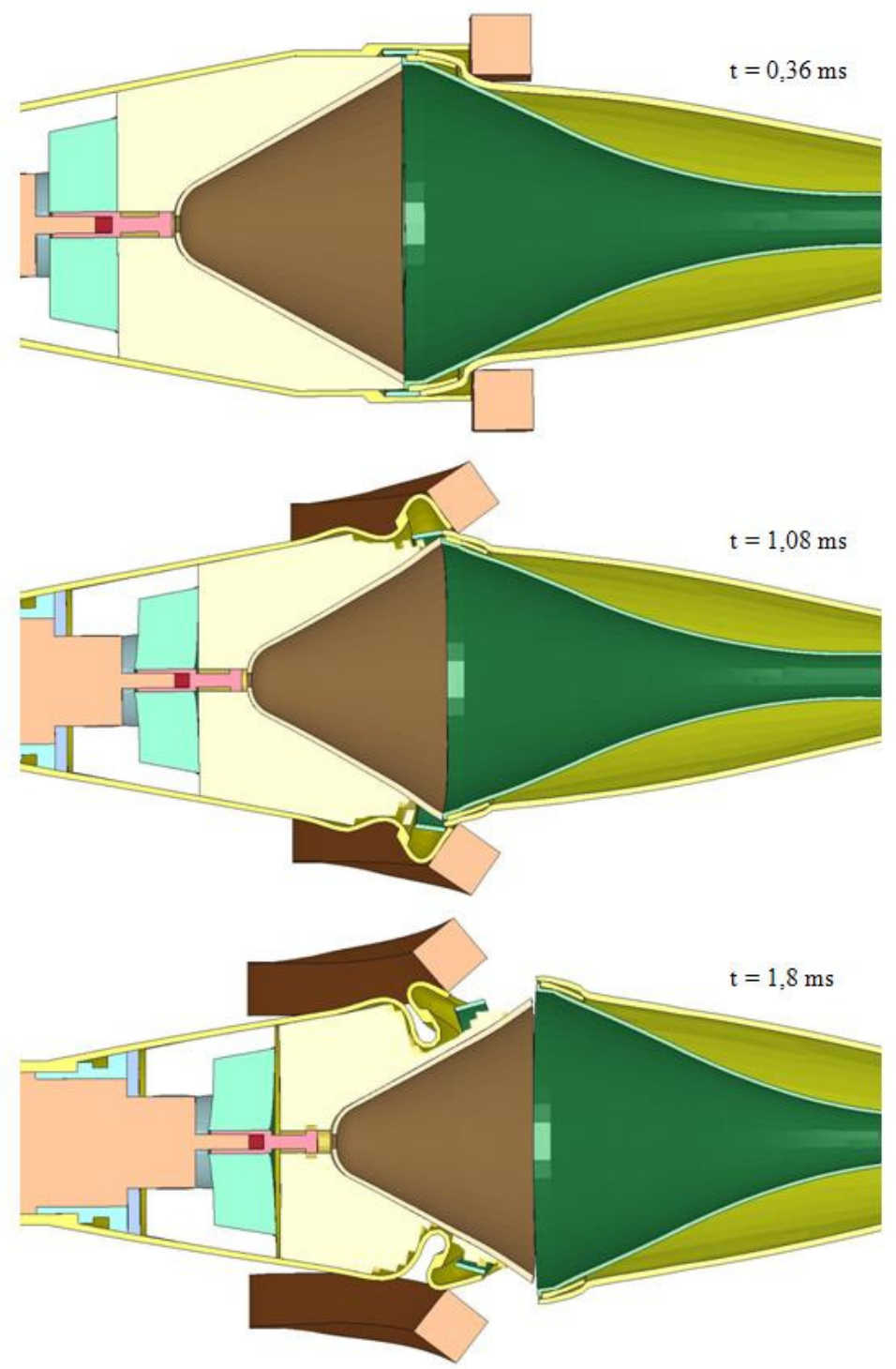

Figure 4: The course of the projectile's deformation for the impact speed $100 \mathrm{~m} / \mathrm{s}$. 

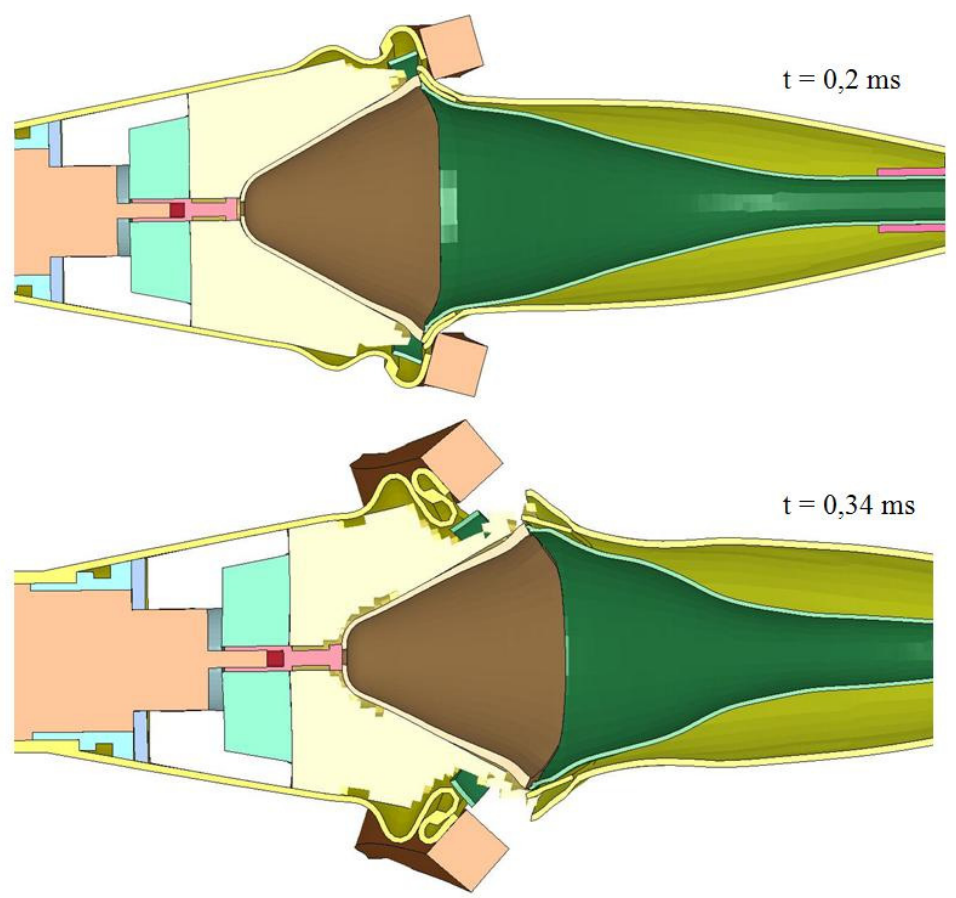

Figure 5: The course of the projectile's deformation for the impact speed 300 $\mathrm{m} / \mathrm{s}$.
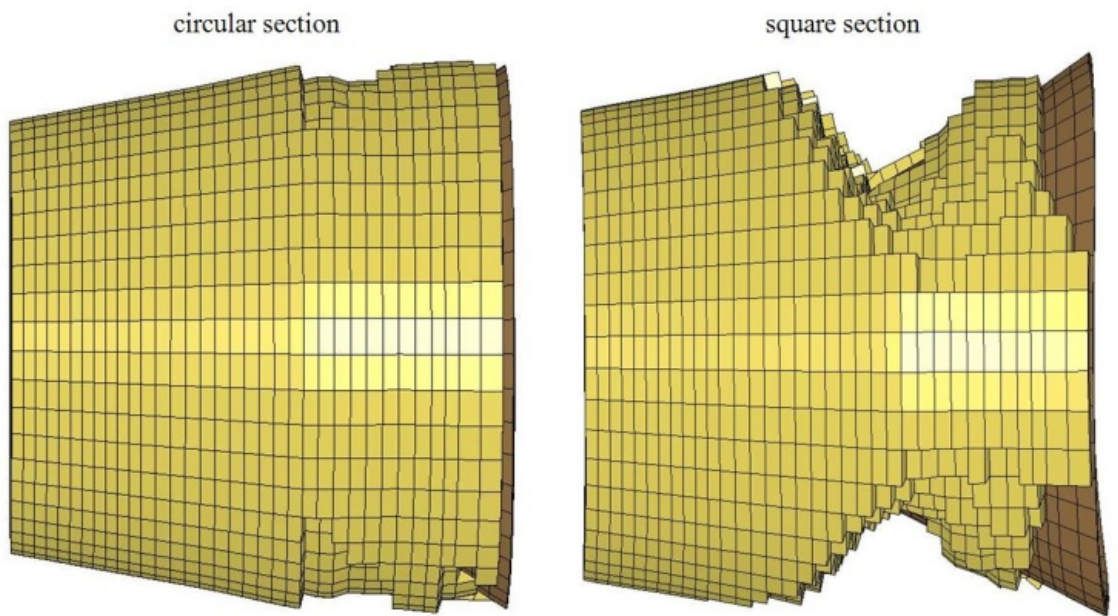

Figure 6: Maximal deformation of the accumulation insert and the destruction of the explosive material for the initial speed of the projectile flight $100 \mathrm{~m} / \mathrm{s}$. 

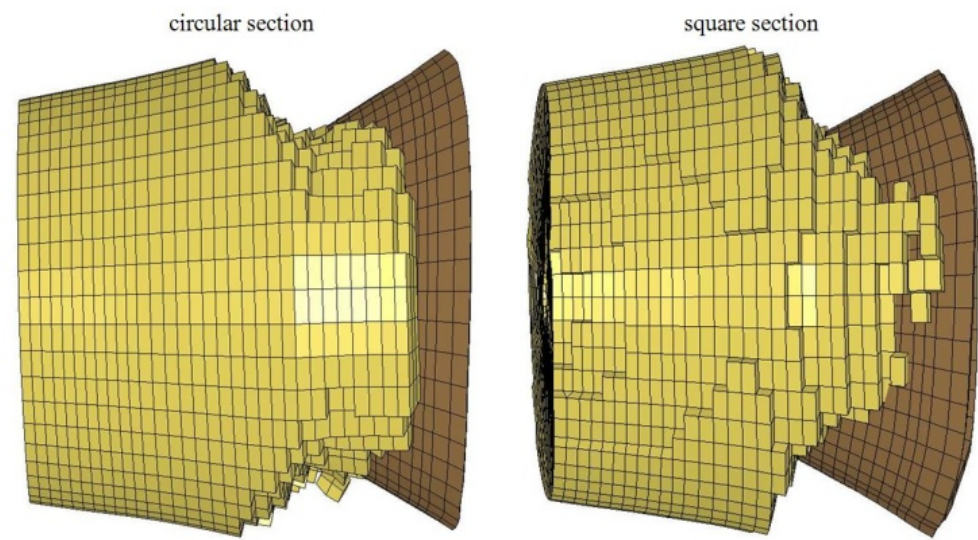

Figure 7: Maximal deformation of the accumulation insert and the destruction of the explosive material for the initial speed of the projectile flight $200 \mathrm{~m} / \mathrm{s}$.
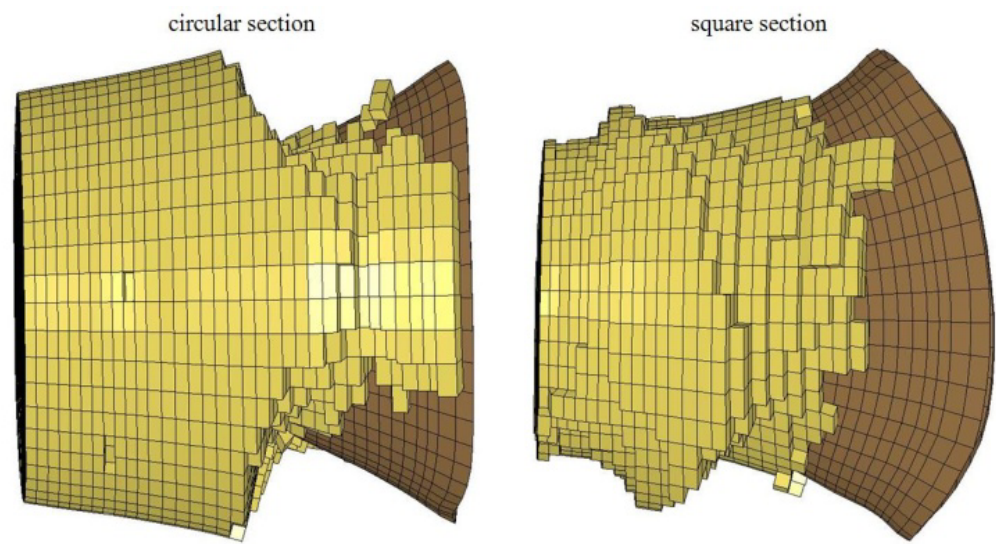

Figure 8: Maximal deformation of the accumulation insert and the destruction of the explosive material for the initial speed of the projectile flight $300 \mathrm{~m} / \mathrm{s}$.

In figures 6, 7 and 8 the maximal destruction of the explosive material and the accumulation insert recorded during the whole simulation, were presented. On the basis of the figures we can observe that the destruction is greater in case of the impact into the rods with a square intersection. Additionally, together with the increase of the initial speed of the projectile, the explosive material is crushed more uniformly on its whole length. 


\section{Conclusions}

From the point of view of protecting the soldiers and the equipment the most important thing is the fact of the creation, or the lack of it, of the accumulation stream. The condition of the explosive material and the accumulation insert at the moment of the fuse actuation decide about the creation of it.

The analysis carried out showed that with the change of the rods crosswise intersection shape (from circular to square) maintaining the same mass of the whole armour, the efficiency of the projectile decreases. The key elements of the projectile, such as the explosive material and the accumulation insert, are deformed more and at the same time have lower ability of creating an accumulation stream which is the greatest destructive power of this type of weapon.

The speed of the projectile during impact with the armour is not without meaning. The increase of the speed causes, before all, the damage of the explosive material on a greater surface.

\section{References}

[1] Wiśniewski A., Pancerze budowa, projektowanie $i$ badanie, WNT, Warszawa, 2001.

[2] www.ruag.com.

[3] Military Parade, Russia, Moscow, 2001.

[4] J.O.Hallaquist: LS-Dyna. Theoretical manual, Livermore Software Technology Corporation, California, 2005. 